(e-migrinter

e-Migrinter

$14 \mid 2016$

L'ajustement méthodologique comme fabrique critique du savoir dans les études migratoires

\title{
S'ouvrir au marché matrimonial globalisé : le cas des femmes russophones
}

\section{Laure Sizaire}

\section{OpenEdition}

\section{Journals}

Édition électronique

URL : https://journals.openedition.org/e-migrinter/706

DOI : 10.4000/e-migrinter.706

ISSN : 1961-9685

Éditeur

UMR 7301 - Migrinter

Référence électronique

Laure Sizaire, "S'ouvrir au marché matrimonial globalisé : le cas des femmes russophones », eMigrinter [En ligne], 14 | 2016, mis en ligne le, consulté le 20 mai 2021. URL : http://

journals.openedition.org/e-migrinter/706 ; DOI : https://doi.org/10.4000/e-migrinter.706

Ce document a été généré automatiquement le 20 mai 2021.

Tous droits réservés 


\title{
S'ouvrir au marché matrimonial globalisé : le cas des femmes russophones
}

\author{
Laure Sizaire
}

\section{NOTE DE L'AUTEUR}

Je remercie particulièrement Jean-Hugues Déchaux pour ses nombreux conseils lors de la rédaction de cet article.

1 L'émission de Striptease, diffusée en 2012, «Berger cherche bergère désespérément », symbolise les représentations communes associées aux mariages contractés entre un homme français, aidé par un intermédiaire (agences, sites internet) et une femme étrangère. Cet épisode nous plonge dans la vie d'une famille paysanne du Pas-de-Calais dont le petit fils n'a pas encore "pris femme ». Sa grand-mère décide alors de faire appel à une agence matrimoniale internationale pour remédier à cette situation et une jeune fille roumaine, ne parlant pas français, se retrouve introduite dans la ferme familiale. L'intérêt de l'émission est de mettre en image les stéréotypes véhiculés sur la pratique des "mariages par correspondance", aussi appelée dans la littérature anglophone, mail order bride, en utilisant la figure d'un paysan au faible potentiel de séduction, mal à l'aise en société et celle d'une très jeune fille, privée de parole, réduite à un statut de marchandise ${ }^{1}$.

2 Cette pratique, bien qu'elle se développe depuis les années 1990, reste en effet encore largement méconnue et stigmatisée. Ces mariages par-delà les frontières impliquent des hommes, généralement issus des pays dits occidentaux, et des femmes de pays émergents ou en voie de développement ${ }^{2}$, qui sont entrés en relation par l'intermédiaire d'une agence, d'une connaissance ou encore via Internet, soit grâce à l'entremise internationale. La pratique des mariages par correspondance a déjà été observée pendant la colonisation, ou lors de mouvements migratoires, entrainant des 
déplacements de femmes qui rejoignaient des hommes sur le nouveau continent ou dans le pays d'accueil ${ }^{3}$. Ces mariages donnaient cependant lieu à des unions endogames et restaient ponctuels tandis que l'entremise internationale a pris aujourd'hui une ampleur globale favorisant des unions exogames.

Cet article souhaite mettre en lumière les femmes russophones engagées dans une démarche de mariage par correspondance. Le terme russophones a été choisi pour réunir les femmes russes, ukrainiennes et biélorusses; d'abord parce qu'elles partagent une langue commune mais surtout dans la mesure où les agences matrimoniales internationales sont particulièrement présentes dans ces trois pays (Hughes, 2004).

Les migrations entrainées par les mariages par correspondance s'inscrivent dans un champ de recherche encore peu exploré dans la littérature francophone: les migrations par le mariage, soit des flux migratoires qui se situent à l'intersection de relations intimes, de projets migratoires et matrimoniaux et souvent aussi de l'usage des Nouvelles Technologies de l'Information et de la Communication (NTIC). Après avoir réalisé un état des lieux des recherches menées sur les migrations par le mariage, je m'attacherai à montrer la dimension fondamentale du genre dans ces flux migratoires et matrimoniaux qui révèlent l'existence d'un marché matrimonial globalisé et genré. Mon analyse portera ensuite plus précisément sur le phénomène des mariages facilités par une agence ou un intermédiaire dans les pays postsoviétiques. À partir d'une enquête qualitative multisituée auprès d'agences matrimoniales internationales et de leurs clientes, je m'appuierai sur les récits de vie de femmes russes, ukrainiennes et biélorusses, migrantes ou en devenir. Le recours à l'usage du concept de «carrières $»^{4}$ (Martiniello, Rea, 2011) permet de reconstruire le parcours de ces femmes et de montrer les interactions entre le projet migratoire et le projet matrimonial : le premier ne précédant pas automatiquement le second.

5 Considérer les prises de décisions et les réajustements qu'opèrent nos protagonistes révèle à la fois leur statut d'actrices et le poids des conditions structurelles dans lesquelles elles évoluent. La mise en lumière des profils de femmes inscrites dans une agence matrimoniale internationale laisse apparaitre une insatisfaction des femmes face aux rapports sociaux de sexe locaux. Ce mécontentement, sans remettre nécessairement en cause les rôles de genre, participe néanmoins au développement d'un marché matrimonial globalisé et genré, médiatisé par les NTIC.

\section{Les migrations par le mariage : un champ de recherche émergent}

6 En 1975, Mirjana Morokvasic fait sortir les femmes migrantes de l'ombre en réalisant un état des lieux sur les migrations féminines. Puis en 1984, elle marque un changement de perspective dans "Birds of passage are also women... ", un article dans lequel elle dénonce une vision passive et essentialiste des femmes migrantes qui les confinait au cadre du regroupement familial et les renvoyait à leur statut de mère ou d'épouse, gardienne de la tradition (voir Oso Casas, 2004 ; Guerry, 2009).

7 Depuis les années 1990, les femmes sont enfin perçues comme des actrices à part entière, pouvant être à l'initiative de la migration, insérées dans les sphères de production et de reproduction sociale. Les figures qui retiennent particulièrement l'attention de ce champ de recherche, à la croisée des études migratoires et des études 
sur le genre, sont celles des femmes migrantes domestiques ou du care, ainsi que les travailleuses du sexe. Cependant, à l'instar de Morokvasic (2011), je soutiens que certaines femmes migrantes continuent de "rester dans l'ombre", à l'image des migrantes par le mariage. Ce type de migration a pu être écarté des intérêts scientifiques au profit des migrations de travail dans la mesure où il fallait s'éloigner des lieux communs attachés traditionnellement au féminin et par conséquent des migrations familiales. Les femmes migrantes ayant trop longtemps été perçues comme suivant leurs maris dans la migration, aborder les migrations de femmes au travers du regroupement familial et du mariage peut apparaître comme un biais immédiat. Or, si le regroupement familial a été jusqu'alors la forme d'immigration principale liée à la famille, les mariages binationaux sont en augmentation depuis les années 1990 (Riano, 2007) : ils impliquent le regroupement conjugal de deux personnes de nationalités différentes qui ont choisi de s'unir dans le mariage. Cette pratique s'écarte ainsi du traditionnel regroupement familial qui résulte d'une union endogame souvent contractée dans le pays d'origine. Migrer par le mariage peut servir des objectifs divers: contourner la fermeture des frontières, voyager, se déplacer (carrière migratoire) et concrétiser un projet matrimonial (carrière matrimoniale). La notion de carrière permet de penser les différents niveaux d'analyse et les étapes successives par lesquels sont passées les individus. Ces étapes sont décisives dans l'évolution des projets individuels et constituent les carrières migratoires et matrimoniales.

8 En France, l'état de la recherche sur les migrations par le mariage montre que le champ a encore été peu investi contrairement à la littérature anglophone et internationale qui s'est emparée du phénomène depuis ces dernières années (voir Kovacs, Melegh, 2007 ; Williams, 2010; Kraler, Kofman, Kohli, Schmoll, 2011; Charsley, 2012). Pourtant, le regroupement familial est aujourd'hui le principal mode d'entrée légal dans les pays européens ainsi qu'aux États-Unis et le « mariage est devenu, plus que jamais, un pilier de l'immigration légale dans l'Union européenne » (Lutz, cité in Kofman, 2000, p. 71).

C'est dans ce contexte que les migrations par le mariage sont devenues la cible de mesures de plus en plus contraignantes depuis 2003 pour aboutir en France par exemple à la loi « Besson » du 16 juin $2011^{5}$ qui légifère notamment sur les « mariages gris ». Derrière de telles lois se dessinent, en creux, une législation de l'amour qui impose aux migrant-es par le mariage une véritable "injonction à l'amour " (Ricordeau, 2014). L'amour dans la migration devient ainsi un objet éminemment politique et commence à retenir l'attention de la recherche ${ }^{6}$. Non seulement certains pays européens mettent en place des lois restrictives exigeant des revenus suffisants pour pouvoir « faire famille » (D'Aoust, 2012 ; Sirriyeh, 2015) mais il faut aussi répondre à la norme de l'amour occidental moderne considérée comme discours de vérité. Elle implique notamment que l'amour et les sentiments se dissocient fondamentalement des intérêts sociaux et économiques (Zelizer, 2005). Cette sommation de répondre à un idéal occidental illustre bien les rapports de domination exercés par les pays occidentaux sur les populations non-occidentales, qui, pour pouvoir accéder à la conjugalité, doivent se conformer à une idéologie dominante portée sur les sentiments.

10 Ces migrations s'inscrivent ainsi dans une période de transformation du paysage migratoire international, avec notamment la chute du mur de Berlin et l'avènement des NTIC, et mettent en exergue l'existence d'une géographie matrimoniale genrée (Constable, 2003). Les mariages mixtes français montrent par exemple que les hommes français se marient largement plus avec les femmes russes que les femmes françaises ne 
se marient avec les hommes russes (Ined, 2013). Les recherches sur le choix du conjoint ont montré que n'importe qui n'épouse pas n'importe qui (Girard, 1957 ; Bozon, Héran, 2000) et que malgré un poids décisionnel de la parenté affaiblie, l'homogamie sociale perdure. Les couples binationaux présentent une forme hétérogame et un biais de genre puisque seuls les hommes français se marient avec certaines femmes étrangères ${ }^{7}$.

Quels sont donc les ressorts qui réunissent ces hommes et ces femmes à l'échelle globale? La littérature actuelle est florissante sur l'expérience migratoire et matrimoniale des femmes généralement issues des pays d'Asie du Sud-Est (Suksomboon, 2011; Ricordeau, 2012), de Chine (Constable, 2003), d'Amérique du sud (Riano, Bagdhadi, 2007 ; Roca, 2011, 2013) et des pays postsoviétiques (Giabiconi, 2005 ; Patico, 2009 ; Heyse, 2010). Ces travaux révèlent l'existence d'un marché matrimonial globalisé, intrinsèquement liés aux NTIC car les rencontres sont souvent médiatisées par le Web. De plus, ils mettent en exergue une cartographie matrimoniale genrée puisqu'ils n'impliquent que des femmes étrangères issues de pays en développement ou émergents et des hommes majoritairement issus de pays occidentaux. Selon plusieurs recherches, les femmes seraient plus « gagnantes » que les hommes dans la migration, notamment en termes de genre (Morokvasic, 2010) dans la mesure où leur position de genre peut se modifier dans le cadre de la migration de manière positive tandis que les hommes voient souvent leur position de genre dominante être ébranlée. Les femmes pourraient donc avoir davantage recours au mariage dans le cadre de la migration ou provoquer un parcours migratoire dans le cadre d'une romance à l'échelle globale. Ces migrations par le mariage posent ainsi la question de l'empowerment des femmes qui accèderaient à plus de liberté et de puissance d'agir en se mariant et en migrant. Cette hypothèse justifierait une prépondérance des femmes dans les migrations par le mariage mais elle retombe en même temps dans les paradigmes classiques de tradition / modernité entrainant une émancipation " automatique " des femmes migrant vers des pays européens. Observer les arrangements qu'implique le choix de migrer et de se marier apparait plus révélateur des imaginaires géographiques, des buts poursuivis et des reconfigurations des rapports de genre (Riano, 2007).

La partie suivante s'attache à montrer que l'entremise internationale peut apparaître comme un moyen stratégique genré utilisé par les femmes à des fins matrimoniales et migratoires - distinction trouble variant selon les territoires -, qui l'inscrit résolument dans le paysage des migrations contemporaines de femmes.

\section{Un marché matrimonial globalisé, genré et stratégique}

13 Si des recherches s'accordent sur le fait que les mariages transnationaux se développent en parallèle à une mobilité internationale grandissante (Kraler et al., 2011), la place des NTIC et spécifiquement du World Wide Web apparaît majeure comme moyen de rencontre et de communication. Le développement d'Internet coïncide avec l'apparition et le développement des agences matrimoniales et des sites de rencontres internationaux. Les espaces qu'ils occupent sur le Web illustrent la globalisation du marché matrimonial mais aussi son caractère genré, notamment à travers les vitrines virtuelles des agences et des sites de rencontre à caractère ethnique.

Plusieurs recherches se sont attachées à décrire les espaces dédiés aux rencontres internationales en vue d'un mariage. Les résultats de l'analyse de ces plateformes sont souvent sans équivoque: les images et les idées véhiculées s'appuient sur des 
stéréotypes de genre et de race (Ricordeau, 2011). Certaines recherches canadiennes et anglophones (Langevin, Belleau, 2000 ; Hughes, 2004) présentent, quant à elles, ce phénomène sous le terme "mail-order bride industry » soit " l'industrie des promises par correspondance ». Suggérant à des degrés divers une association des agences et des sites internet à des réseaux de prostitution ou de trafic humain, ces analyses s'appuient essentiellement sur des faits divers relatant l'histoire d'un mariage arrangé par une agence qui se termine dans des conditions tragiques. Ces recherches donnent ainsi à voir des femmes qui seraient des êtres sans défense, jeunes et inexpérimentées à l'image du terme "promise » (bride) et alimentent l'idée de leur passivité à travers le terme "par correspondance" (mail-order), sémantiquement rattaché à la consommation. Une autre lecture est pourtant possible, notamment en dépassant les vitrines virtuelles, les faits divers et en allant à la rencontre des protagonistes derrière les récits ou les images.

D'autres recherches se sont attachées à placer au cœur de leurs travaux les femmes et les hommes qui se marient grâce à l'entremise internationale (agences, sites ou connaissances), (Constable, 2003, 2006; Patico, 2009; Suksomboon, 2011; Ricordeau, 2012), mettant en lumière les ressorts de ces dynamiques internationales et les motivations des protagonistes. Elles dépassent le seul prisme des systèmes de domination pour penser la capacité d'action des actrices impliquées dans ces rencontres. Elles ont mis en exergue non seulement la diversité des profils de femmes engagées dans une rencontre internationale mais également rompu avec l'idée commune qui associerait ces unions à des mariages de convenance ou à la traite d'être humain. Au contraire, elles montrent des femmes pour la plupart issues des classes moyennes et éduquées qui peuvent aussi subir un déclassement social en se mariant. Leurs carrières migratoires et matrimoniales sont en prise directe avec leur positionnement de genre, de classe et de race. Les réflexions proposées ici s'inscrivent dans la continuité de ces travaux à partir de l'analyse du cas des femmes russophones agissant au sein du « web matrimonial des migrants » (Diminescu et al., 2010).

\section{L'entremise internationale et les femmes russophones}

Les femmes russes et plus largement russophones apparaissent dans les courants migratoires internationaux et particulièrement dans les migrations par le mariage. Plusieurs études anglophones, aussi bien quantitatives (Niedosmyl et al., 2010 ; Levchenko, 2013) que qualitatives (Johnson, 2007; Van Liempt, 2008; Patico, 2009; Heyse, 2010), ont révélé la place importante des femmes russes dans les flux de migrations par le mariage. De la même manière, les mariages entre un homme français et une femme russe sont significatifs en France. En 2013, ils représentent $6 \%$ de la totalité des mariages mixtes impliquant un homme français et une femme étrangère, arrivant ainsi en troisième position derrière les mariages franco-algériens et francomarocains (Ined, 2013). On peut faire l'hypothèse que les NTIC, conjointement à l'entremise internationale effectuée par les agences ou par des connaissances, permettent à ces hommes et à ces femmes d'entrer en relation et ce, depuis les années 1990. La constance du nombre de mariages franco-russes depuis 2005 (voir graphique $\mathrm{n}^{\circ} 1$, source Insee) indique qu'il ne s'agit pas d'un épiphénomène mais bien d'une pratique qui dure. 
Graphique $\mathrm{n}^{\circ} 1$ : Proportion de mariages franco-russes au sein des mariages entre un homme français et une femme étrangère.

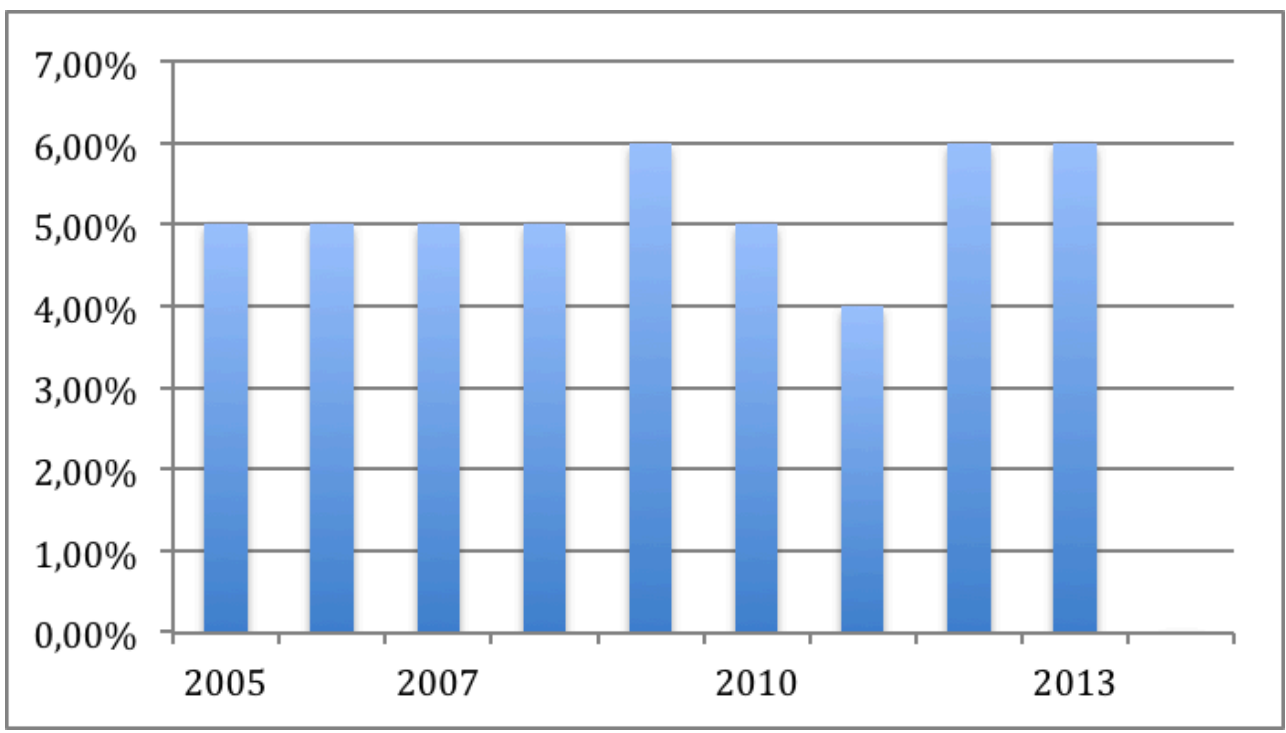

Calculs réalisés par Laure Sizaire à partir des données de l'Insee.

SOURCE : INSEE, « T22 : MARIAgES MIXTES ET MARIAgES ENTRE ÉTRANgERS SELON LE SEXE ET LA NATIONALITÉ DU CONJOINT » (ANNÉE 2005 À 2013).

Mon enquête s'appuie sur des "récits de vie » (Bertaux, 1997) réalisés avec une vingtaine de femmes, russes, ukrainiennes et biélorusses inscrites dans des agences, et sur une ethnographie des agences matrimoniales internationales (Barnaoul, SaintPétersbourg, en Russie; Kiev, Kherson, Kharkov, en Ukraine; Minsk, Grodno, au Belarus). Les premières enquêtes se sont d'abord déroulées en dehors des capitales et des grandes métropoles et ont permis d'accéder aux pratiques des agences dans toute leur diversité et à l'usage qu'en font les femmes russophones. Elles apparaissent comme des structures d'opportunités matrimoniales mais également migratoires et professionnelles spécifiquement genrées, car ce sont essentiellement des femmes qui agissent au sein de celles-ci.

Des temps d'immersion longs dans chaque pays m'ont également permis de réaliser les entretiens en russe et surtout d'accéder à une compréhension empirique de la situation des femmes russophones. Il a aussi été possible de produire des données quantitatives grâce aux bases de données des agences matrimoniales mettant en lumière le profil des adhérentes (âge, situation matrimoniale, maternité, profession, niveau d'études). Cette méthode permet d'embrasser les différents niveaux d'analyse, tant les contraintes et les ressources individuelles que structurelles, et d'envisager ces femmes comme des actrices à part entière tout en reconnaissant «le caractère irréductible de la dualité individu / structure » (Déchaux, 2010, p. 721). Et c'est en articulant les différents niveaux d'analyse qu'il est possible d'envisager ces parcours migratoires, qui parfois sont encore à l'état de projets, comme des " carrières » matrimoniales et migratoires. Dans le cadre des migrations par le mariage, ces carrières se situent au croisement de deux projets : le projet matrimonial et le projet migratoire et dans le cas des femmes russophones qui ont recours à une agence, c'est la carrière matrimoniale qui déclenche la carrière migratoire. 


\section{Un projet avant tout matrimonial}

Les différentes enquêtes effectuées ont permis de distinguer trois catégories de femmes russophones inscrites dans une agence matrimoniale internationale :

(1) Des femmes âgées de 28 à 35 ans sans enfant, issues de la classe moyenne, qui ont pu accéder à des études supérieures; néanmoins, elles ne trouvent pas toujours d'emploi en corrélation avec leur niveau d'étude et cela, cumulé à la difficulté de trouver un conjoint pour fonder une famille, les poussent à élargir leur recherche ;

(2) Des femmes âgées de 35 à 40 ans, sans enfant, issues des classes supérieures, qui, elles, ont pu réaliser une carrière professionnelle mais, parvenues à un certain âge, se retrouvent quasiment exclues du marché matrimonial local; trouver un conjoint et fonder une famille deviennent alors leurs priorités ;

(3) Des femmes de 40 ans et plus, avec au moins un enfant, qui sont plus souvent issues des classes populaires et n'ont pas suivi de longues études ; elles ont déjà expérimenté un mariage et une maternité et ont bien souvent été abandonnées par leur mari. Ayant vécu dans des conditions assez difficiles, elles cherchent à améliorer leurs conditions sociales et économiques pour elles et, parfois, pour leur(s) enfant(s).

À l'image des recherches menées sur les romances à l'échelle globale (Constable, 2003; Patico, 2009; Suksomboon, 2011; Ricordeau, 2012), les femmes qui s'inscrivent dans une agence matrimoniale internationale disposent en grande majorité d'un capital culturel et social élevé. Cependant, un niveau de formation et de qualification supérieure ne s'accompagne pas forcément d'une situation économique favorable. À la chute du régime soviétique, les professions intellectuelles du secteur d'État ont subi une détérioration importante de leur niveau de vie et malgré des positions sociales élevées, les salaires peuvent être extrêmement bas. Les femmes apparaissent ainsi dans les groupes sociaux les plus vulnérables dans la mesure où «dans la majorité des branches d'activité, les salaires féminins sont inférieurs de plus d'un tiers aux rémunérations masculines » (Mespoulet, 2005, p. 188). Contrairement à la France où il est possible d'envisager, pour les femmes d'un niveau d'éducation élevé accompagné d'une réussite professionnelle, d'accéder à une autonomie confortable, les pays étudiés n'offrent souvent pas cette opportunité (Lebedev, 2007). Ces quelques remarques sont nécessaires pour mieux appréhender la situation des femmes russophones et leurs attentes vis-à-vis du mariage. Alors que cette institution a perdu de sa centralité dans les pays occidentaux depuis les années 1970 face à l'évolution majeure de la place des femmes (Déchaux, 2009), elle reste fondamentale dans les pays russophones.

De la «working-mother» qui prévalait sous le régime soviétique, impliquant l'accomplissement des devoirs civiques dans la sphère professionnelle et de leur destinée dans la maternité, cette triple charge (professionnelle, domestique et reproductive) va peu à peu être remise en cause pour aboutir, dans les années 1990, à un nouveau discours hégémonique à la fois populaire et politique reposant sur l'idéal $\mathrm{du}$ rôle de l'homme, pourvoyeur économique et de la femme, gardienne du foyer (Temkina, Rotkirch, 1997). La grande majorité des femmes interrogées avaient visiblement intégré ce modèle :

L'homme a deux travails et sa femme s'occupe des enfants, prépare le diner. Dans les familles russes, c'est normal mais quand vous êtes seule, vous faites le diner, le ménage, et 
vous devez rapporter l'argent. (Inna, 41 ans, mariée à un homme français, mère d'un enfant adulte issu d'un précédent mariage en Russie, 2012). politiques natalistes russes qui poussent les femmes à avoir plus d'enfants et à rester au foyer pour s'occuper d'eux (Rotkirch et al., 2007). Dans cette optique, se marier et avoir des enfants reste une norme prégnante pour les femmes et la maternité continue à être un élément crucial de leur identité (Temkina, 2010, p. 97). Mais l'âge socialement requis pour entrer dans le mariage et la maternité se situe entre 20 et 25 ans selon les femmes interrogées. Au-delà de 30 ans, elles subissent une forte marginalisation sur le marché matrimonial local (Patico, 2009; Heyse, 2010). Elles n'hésitent pas à se désigner ellesmêmes comme de "vieilles femmes » passé cet âge. En cela, elles rejoignent les femmes engagées dans le marché matrimonial globalisé. Les femmes philippines, thailandaises, chinoises sont aussi confrontées à cette stigmatisation (Constable, 2003 ; Suksomboon, 2011 ; Ricordeau, 2012). Le second point similaire est la critique qu'elles portent sur les hommes de leur pays. Les femmes postsoviétiques sont confrontées à un déséquilibre démographique homme / femme important, en particulier dans certaines régions comme la Sibérie occidentale (Barnaoul), le sud-est de l'Ukraine (Kherson) et le nordouest du Belarus (Grodno). Cette différence d'effectif ${ }^{8}$ est notamment due à une mobilité masculine plus importante, à la perte d'hommes pendant les conflits en Tchétchénie et en Afghanistan, mais plus encore à la «crise de la condition masculine ", largement décrite dans la littérature sur les masculinités russes (Rotkirch, 2000 ; Ashwin, Lytkina, 2004). Il s'agit des difficultés que beaucoup d'hommes russes ont rencontré pour faire face au passage extrêmement rapide d'une économie planifiée à une économie de marché, où nombre d'entre eux ont perdu leur emploi et, par conséquent, ne pouvaient plus assumer leur seul et unique rôle dédié: celui de breadwinner. L'espérance de vie chez les hommes chute ainsi considérablement, en passant de 64,5 ans en 1964 à 58,5 ans en 2002 (Blum, 2005, 153-154). En 2006, elle est encore extrêmement éloignée de celle des femmes, à savoir, 60,5 ans contre 74 ans. La situation des hommes devient une préoccupation nationale et féminine (Luehrmann, 2004), au même moment où se développent massivement les agences matrimoniales internationales.

\section{À la recherche d'un homme de « bonne qualité »}

Les directrices des agences matrimoniales internationales postsoviétiques soutiennent que leurs adhérentes cherchaient dans les années 1990 à fuir le pays, alors en proie à une crise majeure dont les femmes étaient les premières touchées. Elles expliquent aujourd'hui que la plupart de leurs clientes sont à la recherche "de l'amour", de la "perle rare ", de leur « moitié ». Les attentes associées à la recherche d'un partenaire varient toutefois considérablement selon les normes sociales en vigueur. Comme nous l'avons déjà évoqué, les rôles et les identités de genre se sont modifiés durant les années 1990 dans les pays postsoviétiques et l'une des revendications principales des femmes de notre enquête est de n'avoir accès qu'à des hommes mariés ou de « mauvaise qualité ». Le point de vue des femmes ukrainiennes ou biélorusses n'est pas aussi tranché mais leurs trajectoires amoureuses et familiales montrent qu'elles ont aussi été confrontées à des problèmes d'alcoolisme, d'abandon et parfois de violence. Les discours se rejoignent cependant dans la quête d'un «homme bien", qui pourrait

e-Migrinter, 14 | 2016 
être un «mari sérieux» (Patico, 2009). La "mauvaise qualité » des hommes dont parlent les femmes est directement liée au discours sur la "crise de la condition masculine" des hommes russes. Elles partagent une connaissance commune des hommes qui rattachent ces derniers à des comportements tels que l'alcoolisme, l'abandon, les violences domestiques, le manque de soutien et l'infidélité. Toutefois, il ressort aussi des discours la recherche d'autres rapports avec les hommes qui, dans les régions les plus touchées par le déséquilibre démographique, sont décrits comme ayant le choix :

À Grodno, les hommes ont le choix. Ils peuvent choisir et ils ne sont donc pas attentionnés, c'est la femme qui doit être aux petits soins si elle veut le garder. (Yulia, 29 ans, Grodno, Bélarus, 2014).

\section{Avoir (aussi) le choix}

S'ouvrir au marché matrimonial globalisé, c'est donc aussi s'ouvrir à de nouvelles opportunités. La plupart des femmes qui s'inscrivent dans une démarche de rencontre internationale ont des amies ou des connaissances déjà mariées à l'étranger qui symbolisent la «réussite » d'un projet matrimonial et migratoire et l'efficacité des agences.

J'ai une amie qui s'est mariée à l'étranger, elle a un mariage heureux. Je cherche un homme bien, à l'étranger ou ici. Depuis le début, je cherche des deux côtés, là où ça peut marcher. (Marina, 42 ans, célibataire, sans enfant, Barnaoul, 2012).

La popularité et le développement des agences matrimoniales s'expliquent par le bouche à oreille. Les femmes ne tombent pas par hasard sur ces structures, elles les connaissent depuis longtemps par leur réputation. Le choix d'y avoir recours a été mûrement réfléchi et s'inscrit à la suite d'une trajectoire matrimoniale avec des hommes russes. Il n'est pas rare que les mères conseillent à leur fille de s'inscrire dans une agence matrimoniale internationale (Hughes, 2004) si elles ne sont pas déjà inscrites elles-mêmes. C'est à travers ces différentes étapes qu'apparaît la carrière matrimoniale de ces femmes. L'utilisation des agences peut ainsi être véritablement perçue comme un outil mobilisé par les femmes en vue de réaliser leurs propres objectifs, fonder une famille avec un mari sérieux, lesquels sont néanmoins en partie modelés par la socialisation de ces femmes. Zdravomyslova et Temkina (2006) ont fait ressortir deux types de féminité " néo-traditionnaliste » dans les pays postsoviétiques : le modèle de la « mère travailleuse » (working mother) et celui de la « femme au foyer ». Le premier est devenu un choix personnel, détaché des obligations civiles et le second est perçu comme un avenir attrayant dans la mesure où cette position est rarement possible, et dans les classes supérieures, comme une marque de statut social (Zdravomyslova \& Temkina, 2006, p. 110).

Ces modèles apparaissent nettement dans le récit de ces femmes : avoir le choix de travailler "pour le plaisir", pouvoir se former, apprendre des langues, des pratiques professionnelles mais sans avoir à se préoccuper de «subvenir» aux besoins de la famille, qui doit être une prérogative de l'homme. 
Je veux travailler mais pour le plaisir, comme un hobby. J'aime bien travailler sur

l'ordinateur. Mais le but de ma vie, c'est être mère. Pour le moment oui. (Tatiana, 29 ans, célibataire, Kherson, Ukraine, 2013).

Être femme au foyer peut ainsi être une perspective tout à fait attrayante et souhaitée dans la mesure où ce choix n'existe pas. Alors que le féminisme de la seconde vague des années 1970 en France prônait une libération des femmes par le travail, au même moment pour les femmes russophones, la libération, c'est aussi de pouvoir choisir. La recherche de l'amour ou de la "perle rare" est ainsi intrinsèquement liée à la socialisation et au poids de l'histoire partagés par ces femmes. Un homme de «bonne qualité ", c'est avant tout un homme qui ne boit pas et qui subvient aux besoins de la famille. Cette représentation entre en résonnance avec celle des femmes à l'époque soviétique qui déjà décrivaient un "good enough husband" comme un homme qui ne boit pas, gagne suffisamment, ne dépense pas tout en cigarettes et boissons, qui participe à la maison et qui n'est pas brutal au lit (Rotkirch, 2000).

\section{Conclusion}

31 Ainsi, les femmes inscrites dans des agences matrimoniales internationales poursuivent un projet qui apparait avant tout matrimonial et qui s'apparente à une "carrière »: d'une part, par les différentes étapes qui jalonnent le parcours de ces femmes et qui provoquent nombre de changements, de prises de décisions ainsi que la mobilisation de différents réseaux et d'autre part, devant l'injonction postsoviétique de devenir une mère, une épouse. Une carrière matrimoniale dont découle, le cas échéant, une carrière migratoire. Se donner plus de choix et avoir plus d'opportunités, inscrit donc résolument ces femmes dans une démarche d'actrice. Mais il est clair, dans le même temps, qu'elles sont prises dans des conditions structurelles genrées fortement inégalitaires et étriquées : se marier reste donc un moyen d'autonomisation selon des objectifs socialement construits. Alors que la littérature sur les migrations par le mariage souligne souvent, chez les femmes, la recherche d'autres rapports de genre dans un désir d'émancipation (Riano, Bagdhadi, 2007 ; Ricordeau, 2012), cet aspect est à nuancer à la lumière du discours des femmes que j'ai rencontrées : elles ne cherchent pas un mariage qui impliquerait de tout nouveaux fondements dans les rapports de genre mais elles montrent néanmoins qu'elles souhaitent accéder à plus de choix et rejettent le sort qui leur est réservé dans leur pays.

La globalisation du marché matrimonial, rendu possible avec l'avènement des NTIC, cristallise ainsi des tensions dans les rapports sociaux de sexe internationaux. Les protagonistes des mariages facilités par des agences sont des femmes dont les contextes socioculturels et historiques varient largement, et pourtant, les analyses ethnographiques montrent des similitudes dans leurs démarches respectives. Souvent marginalisées sur le marché matrimonial local, elles portent un regard critique sur les hommes locaux et se tournent vers l'international pour accéder à d'autres choix matrimoniaux, des opportunités migratoires ou encore professionnelles (Constable, 2003 ; Patico, 2009 ; Ricordeau, 2012). Elles s'engagent ainsi dans une carrière à la fois matrimoniale et migratoire, développant des stratégies en mobilisant les ressources dont elles disposent, quand bien même celles-ci sont souvent l'origine ou la conséquence des rapports de domination qu'elles subissent: leur sexe, les agences 
matrimoniales et les sites de rencontres internationaux. Ce qui rejoint un discours hégémonique porté sur la sexualité des femmes russes qui est présentée comme l'un de leur principal atout de réussite dans la vie, pouvant être "échangée contre des bénéfices sociaux et du pouvoir d'achat, signe d'un «good deal » (Zdravomyslova \& Temkina, 2006, p. 110).

Ces femmes migrantes ou en devenir s'appuient ainsi sur l'ordre établi pour poursuivre leurs propres objectifs à l'image des femmes migrantes (Morokvasic, 2010). Ces premiers résultats montrent des femmes qui répondent à des normes de genre globalisées et commercialisées par les agences matrimoniales ou les sites de rencontre, mais qui désirent aussi accéder à d'autres opportunités matrimoniales à l'échelle globale. Poursuivre des recherches auprès des couples franco-russes semble nécessaire pour comprendre les nouveaux "arrangements entre les sexes" (Goffman, 2002) à l'échelle globale et répondre à la question de l'empowerment des femmes insérées dans les flux de migration par le mariage.

\section{BIBLIOGRAPHIE}

Ashwin, Sarah; Lytkina, Tatyana (2004) Men in Crisis in Russia: The Role of Domestic Marginalization, Gender \& Society, vol. 18, n², pp. 189-206.

Bertaux, Daniel (2005) Le récit de vie, Paris, Armand Colin, 128 p.

Blum, Alain (2005) Individu, famille et population en Russie, in Rey, M.-P. (Dir.) Les Russes de Gorbatchev à Poutine, Paris, Armand Colin, $240 \mathrm{p}$.

Charsley, Katharine (Dir.) (2012) Transnational Marriage: new perspectives from Europe and beyond, New-York, Routledge, $250 \mathrm{p}$.

Constable, Nicole (2003) Romance on a global stage, Berkeley, University of California Press, 293 p.

Constable, Nicole (2006) Brides, maids, and prostitutes: reflections on the study of 'trafficked' women, Journal of Multidisciplinary International Studies, vol. 3, n 2 [réf. du 5 juillet 2015] [Disponible sur Internet].

D’Aoust, Anne-Marie (2012) Les couples mixtes sous haute surveillance, Plein droit, $\mathrm{n}^{\circ}$ 95, pp. 15-18.

Déchaux, Jean-Hugues (2009) Sociologie de la famille, Paris, La Découverte, 128 p., (Repères).

Déchaux, Jean-Hugues (2010) Agir en situation : effets de disposition et effets de cadrage, Revue française de sociologie, vol. 51, $\mathrm{n}^{\circ} 4$, pp. 720-746.

Diminescu, Dana ; et alli (2010) Le web matrimonial des migrants. L'économie du profilage au service d'une nouvelle forme de commerce ethnique, Réseaux, $\mathrm{n}^{\circ} 159$, pp. 15-56.

Giabiconi, Dominique (2005) Les mariages mixtes franco-polonais, Revue européenne des migrations internationales, vol. 21, $\mathrm{n}^{\circ} 1$, pp. 259-273.

Goffman, Erving (2002) L'arrangement des sexes, Paris, La dispute, 115 p. 
Guerry, Linda (2009) Femmes et genre dans l'histoire de l'immigration. Naissance et cheminement d'un sujet de recherche, Genre \& Histoire, $n^{\circ} 5$ [réf. du 5 juillet 2015] [Disponible sur Internet].

Heyse, Petra (2010) Deconstructing Fixed Identities: An Intersectional Analysis of Russianspeaking Female Marriage Migrants' Self-representations, Journal of Intercultural Studies, vol. 31, $\mathrm{n}^{\circ} 1$, pp. 65-80.

Hughes, Donna (2004) The Role of "Marriage Agencies" in the Sexual Exploitation and Trafficking of Women from the Former Soviet Union, International Review of Victimology, vol. 11, $\mathrm{n}^{\circ} 1$, pp. 49-71.

INSEE (2013) T22: Mariages mixtes et mariages entre étrangers selon le sexe et la nationalité du conjoint. Année 2013 [réf. du 5 juillet 2015] [Disponible sur Internet]

Johnson, Ericka (2007) Dreaming of a Mail-Order Husband, Durham, Duke University Press, 208 p.

Kofman, Eleonore; Phizackela, Annie; Raghuram, Parvati; Sales, Rosemary (2000) Genre and International Migration in Europe: Employment, Welfare and Politics, London; New York, Routledge, $234 \mathrm{p}$.

Kovacs, Eva; Melegh, Attila (2007) In a gendered space. Forms and Reasons of migration and integration of female migrants, Demográfia, vol. 50, n 5, English Edition, pp. 26-59.

Kraler, Albert; Kofman, Eleonore; Kohli, Martin; Schmoll, Camille (Dir.) (2011) Gender, Generations and the Family in International Migration, Amsterdam, Amsterdam University Press, 416 p. (IMISCOE Research).

Langevin, Louise ; Belleau, Marie-Claire (2000) Le trafic des femmes au Canada : une analyse critique du cadre juridique de l'embauche d'aides familiales immigrantes résidantes et de la pratique des promises par correspondance, Ottawa, Condition féminine du Canada, $220 \mathrm{p}$.

Lebedev, Anna (2007) Femmes en Russie : une inégalité qui ne dit pas son nom, Après-demain, $n^{\circ} 2$, pp. 5-8 (Le statut des femmes dans le monde, II) [Disponible sur Internet].

Levchenko, Polina; Solheim, Catherine (2013) International Marriages between Eastern European-Born women and US born men, Family Relations, $n^{\circ}$ 62, pp. 30-41.

Luehrmann, Sonja (2004) Mediated Marriage: Internet Matchmaking in Provincial Russia, EuropeAsia Studies, vol. 56, $\mathrm{n}^{\circ}$ 6, pp. 857-876.

Martiniello, Marco ; Rea, Andrea (2011) Des flux migratoires aux carrières migratoires, SociologieS, (Migrations, pluralisation, ethnicisation des sociétés contemporaines) [réf. du 5 juillet 2015] [Disponible sur Internet]

Mespoulet, Martine (2005) Des différences sociales accrues, in Rey, M.-P. (Dir.) Les Russes de Gorbatchev à Poutine, Paris, Armand Colin, $240 \mathrm{p}$.

Morokvasic, Mirjana (1984) Birds of Passage Are Also Women..., International Migration Review, vol. $18, \mathrm{n}^{\circ} 4$, pp. 886-907.

Morokvasic, Mirjana (2010) Le genre est au cœur des migrations, in Falquet, J. ; et alli (Dir.) Le sexe de la Mondialisation, Paris, Presses de Sciences Po, pp. 105-120 (Académique).

Morokvasic, Mirjana (2011) L’(in)visibilité continue, Cahiers du Genre, vol. 51, n 2, pp. 25-47. Niedomysl, Thomas; Östh, John; Van Ham, Maarten (2010) The Globalisation of Marriage Fields: The Swedish Case, Journal of Ethnic and Migration Studies, vol. 36, n 7, pp. 1119-1138. 
Oso Casas, Laura (2004) Femmes, actrices des mouvements migratoires, in Reysoo, F. ; Verschuur, C.(Dir.) Femmes en mouvement. Genre, migrations et nouvelle division internationale du travail, Berne ; Genève, DDC/Unesco/iuéd, pp. 165-193.

Patico, Jennifer (2009) For love, money, or normalcy: Meanings of strategy and sentiment in the Russian- American matchmaking industry, Ethnos, n 74, pp. 307-330.

Riano, Yvonne ; Baghdadi, Nadia (2007) “Je pensais que je pourrais avoir une relation plus égalitaire avec un Européen". Le rôle du genre et des imaginaires géographiques dans la migration des femmes, Nouvelles Questions Féministes, vol. 26, nº 1, pp. 38-53.

Ricordeau, Gwenola (2011) À la recherche de la femme idéale... Les stéréotypes de genre et de race dans le commerce de "promises par correspondance ", Genre, Sexualité et Société, $\mathrm{n}^{\circ}$ 5, [réf. $\mathrm{du} 5$ juillet 2015] [Disponible sur Internet].

Ricordeau, Gwenola (2012) Devenir une First World Woman : stratégies migratoires et migrations par le mariage, Sociologies, [réf. du 5 juillet 2015] [Disponible sur Internet].

Ricordeau, Gwenola (2014) Des femmes "mariées par correspondance" aux mariages "gris" : L'injonction à l'amour faite aux femmes du Sud, 1er Congrès Études de Genre en France, Institut du genre ; ENS, Lyon, 3-5 septembre 2014.

Roca, Jordi (2011) (Re)buscando el amor : Motivos y razones de las uniones mixtas de hombres españoles con mujeres extranjeras, Revista de Dialectología y Tradiciones Populares, vol. LXVI, $\mathrm{n}^{\circ} 2$, pp. 487-514.

Roca, Jordi (2013) Les familles mixtes en Espagne. Liens transnationaux et processus d'intégration, Diversité Urbaine, vol. 13, n², pp. 29-48.

Rotkirch, Anna (2000) The Man Question Loves and Lives in Late 20th Century Russia, Helsinski, University of Helsinki; Department of Social Policy, 339 p. (Research Reports).

Rotkirch, Anna; Temkina, Anna; Zdravomyslova, Elena (2007) Who helps the degraded housewife? Comments on Vladimir Putin's demographic speech, European Journal of Women's Studies, $\mathrm{n}^{\circ} 14$, pp. 349-357.

Sirriyeh, Ala (2015) 'All you need is love and $£$ 18,600': Class and the new UK family migration rules, Critical Social Policy, vol. 35, $\mathrm{n}^{\circ} 2$, pp. 228-247.

Suksomboon, Panitee (2011) Cross-broder marriage as a migration strategy: Thai women in the Netherlands, in Kraler, A.; Kofman, E.; Kohli, M.; Schmoll, C. (Dir.) Gender, Generations and the Family in International Migration, Amsterdam, Amsterdam University Press, 416 p. (IMISCOE Research).

Temkina, Anna (2010) Childbearing and Work-Family Balance among Contemporary Russian Women, Finnish Yearbook of Population Research, vol. XLV, pp. 83-101.

Temkina, Anna; Rotkirch Anna (1997) Soviet Gender Contracts and Their Shifts in Contemporary Russia, in Temkina, A. (Dir.) Russia in Transition: The Case of New Collective Actors and New Collective Actions, Helsinki, Kikimora Publications, 270 p. (Series A1).

Van Liempt, Ilse (2008) Gendered borders: The case of 'illegal' migration from Iraq, the Horn of Africa and the former Soviet Union to the Netherlands, in Schrover, M.; Van der Leun, J.; Lucassen, L.; Quispel, C. (Dir.) Illegal Migration and Gender in a Global and Historical Perspective, Amsterdam, Amsterdam University Press, pp. 83-100. (IMISCOE).

Williams, Lucy (2010) Global marriage: cross-border marriage migration in global context, Basingstoke, Palgrave Macmillan, $264 \mathrm{p}$. 
Zdravomyslova, Elena; Temkina, Anna (2006) Gendered citizenship in Soviet and Post-Soviet societies, in Tolz, V.; Booth, S. (Dir.) Nation and Gender in Contemporary Europe, Manchester, Manchester University Press, pp. 96-113.

Zelizer, Viviana (2001) Transactions intimes, Genèses, n 42, pp. 121-144.

\section{NOTES}

1. Dans la même optique, le film Je vous trouve très beau (2005), d'Isabelle Mergault, s'appuie sur les mêmes stéréotypes.

2. Les pays les plus représentés sont les pays post-soviétiques dont la Russie, l'Ukraine et le Belarus; l'Asie du Sud-Est et en particulier les Philippines et la Chine, et dans une moindre mesure certains pays d'Afrique et d'Amérique du Sud.

3. Par exemple les Picture brides.

4. Le concept de « carrière » est employé dans cet article comme un outil permettant de saisir à la fois les caractéristiques de l'individu pensé comme un acteur et ses interactions avec les structures et les réseaux, pensés comme autant de contraintes et d'opportunités (Martiniello, Rea, 2011).

5. Loi n²011-672 du 16 juin 2011 relative à l'immigration, à l'intégration et à la nationalité.

6. Plusieurs journées d'études ont manifesté de l'intérêt pour cette dimension, notamment «Migration, Amour et État : un ménage à trois?» Université Libre de Bruxelles (Octobre, 2014); «Of Love and Family, States and Borders : Comparative Perspectives on Afro-European Couples and Families », panel 2 « Regulating Love », The University of Chicago, Center in Paris (Décembre 2014).

7. Par exemple, en 2013, 35 mariages entre une femme française et un homme russe ont eu lieu contre 962 entre un homme français et une femme russe (Ined, 2013).

8. La Russie, l'Ukraine et le Belarus ont presque le même ratio homme / femme, soit $54 \%$ de femmes contre $46 \%$ d'hommes à l'échelle du pays (2010) et les zones citées présentent donc une différence d'effectif supérieure aux chiffres nationaux, [réf. du 5 juillet 2015] : source GEOHIVE, [Disponible sur Internet].

\section{INDEX}

Mots-clés : femmes, mariage mixte, comportement matrimonial, filière migratoire Index géographique : Russie, Ukraine, Biélorussie

\section{AUTEUR}

\section{LAURE SIZAIRE}

Doctorante en Sociologie - Anthropologie, Université Lyon 2 (Centre Max Weber)

laure.sizaire@gmail.com 